\title{
Noise Generation of a Bionic Airfoil Based on Owl Wings
}

\author{
Changjiang $\mathrm{Ge}^{1, \mathrm{a}}$ \\ ${ }^{1}$ College of Automotive Engineering, Jilin University, Changchun, 130022, P. R. China
}

\begin{abstract}
A bionic airfoil is used to investigate a preliminary understanding of the noise mechanism generated by the flow around the owl wing. The flow indicates a transitional separation bubble on the surface and the turbulent boundary layer reattaches at the downstream end of the bubble. This causes turbulent boundary layer trailing-edge scattering noise corresponding to the broadband noise of owl wings at low Reynolds numbers. Furthermore, a large-scale vortex detaches from the bubble and then drifts downstream. Thus, a tone will be generated by the vortex shedding. However, the computations of the acoustic field for both cases, where the on-body data surface and the off-body data surface are used respectively, predominantly verify the broadband nature of the noise that well agrees with the noise scattering caused by the turbulent boundary layer passing by the sharp trailing edge. Furthermore, the significant decrease of the spectra around $2 \mathrm{k} \mathrm{Hz}$ is consistent with that of silent owl wings. This fact suggests that the numerical simulations are capturing the basic physical mechanism responsible for the noise radiated by the natural owl wing. This might be able to provide a reference for engineering applications of the owl technology.
\end{abstract}

\section{Introduction}

"Owl technology" is a promising means of turbulent boundary layer trailing-edge noise reduction for applications such as fans, rotors and propellers [1 4], so bionic treatments, inspired by three wings and plumage: leading-edge serrations, trailing-edge fringes and a soft and elastic downy upper surface of the feathers [5], are used to attenuate noise caused by trailing-edge scattering. Furthermore, howe [6 7], using aeroacoustic theory, showed how serrated or porous trailing edge may be made quieter than a straight and solid trailing edge. Despite this accumulated knowledge of airfoil trailing-edge noise, actually alleviating it is difficult. Particularly, experimental results of full-scale models associated with trailing-edge serrations showed noise abatement in the low frequency band, but noise enhancement in the high frequency band [8]. According to howe's theory, significant noise reductions should be only expected in the high frequency region. This suggested that the trailing-edge noise reduction problem is complex, and involves a variety of opposing constrains.

Previous investigations were conducted by a flat plate or technical airfoils [9 13], such as NACA airfoils. Obviously, they are different from profiles extracted by nature owl wings [14 15]. It is well known that modification of the noise-producing characteristics of the trailing-edge turbulent boundary layer is possible through airfoil shape. This may be the reason for the discrepancy between the experiments and theory of noise reduction with the bionic treatments. In addition, Reynolds number of

\footnotetext{
${ }^{\mathrm{a}}$ Corresponding author : gcj@jlu.edu.cn
} 
less than $2 \times 10^{5}$ typical of flying owls implies the laminar flow on the surfaces of owl wings. However, measurements suggested that the pronounced broadband noise is generated by turbulent boundary layer scattering [16]. Thus, fundamental researches are needed to shed more additional light on the owl-based airfoil noise mechanism for further investigations of noise reduction technology.

The objective of this numerical study is to gain a preliminary understanding of the aerodynamic noise mechanism generated by the unsteady flow around a bionic owl wing airfoil. The bionic airfoil selected by the validity is representative of the characteristics of owl wings [17]. The flow field around the airfoil is simulated by Computational Fluid Dynamics (CFD), and the noise source is identified by the distribution of vorticity. The far-field Sound Pressure Level (SPL) is determined by inputting highly resolved unsteady near-field data obtained from converged CFD solutions into twodimensional Ffowcs Williams and Hawkings equation (FW-H equation) in order to estimate noise levels at a radial distance of ten chords from the airfoil.

\section{Materials and method}

\subsection{Grid topology}

The computational domain of the bionic airfoil extends to 20c in the upstream direction, $24 \mathrm{c}$ in the downstream direction and 30c in the crosswise direction so as to dissipate reflections from the boundaries (Figure 1). The leading edge is located at the origin. The outflow boundary condition is a pressure far field, thus minimize effectively any feedback effect in the incompressible flow regime, the viscous wall boundary condition is specified for the bionic airfoil surface, and interface boundary condition is applied at the internal faces. Quadrilateral grids with C-type are employed for the simulation because they generate less numerical diffusion than triangular grids. Typical grids used for the two-dimensional simulations are $250 \times 200$ (Figure 2). The grid size $\Delta \mathrm{x}$ is small enough to capture the interesting turbulence length scales, and to make the numerical diffusion smaller than the subgrid-scale turbulence viscosity. Further node savings are achieved by specifically clustering nodes near the wall. For instance, the node distribution at the wall yields non-dimensional wall distances $\mathrm{y}^{+}<0.4$ everywhere and it can simulate the effect of laminar sub-layer. Moreover, the sufficiently dense grids around the areas of interest are also required to ensure adequate propagation of waves and restrict artificial diffusion.

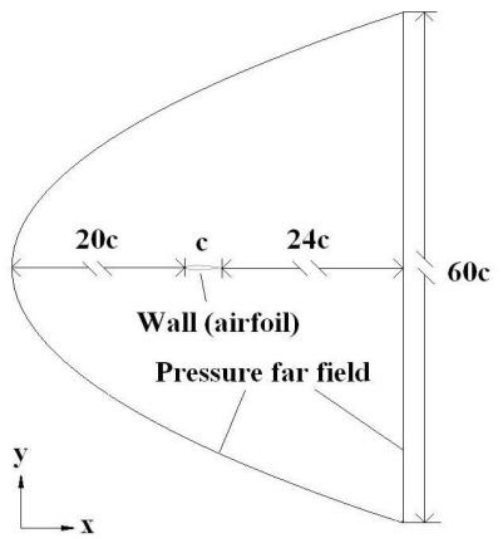

Figure 1. Schematic diagram of the computational domain, c represents the chord length of $0.1 \mathrm{~m}$. 


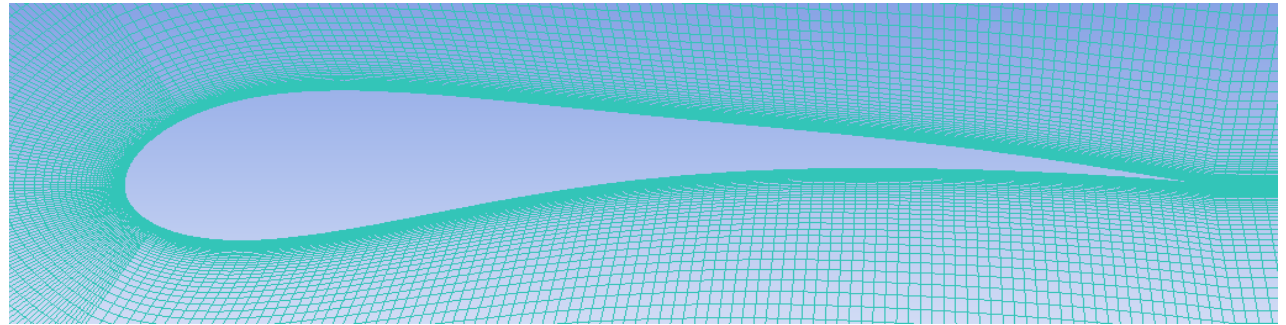

Figure 2. Grid distribution around the bionic airfoil.

\subsection{Numerical method}

The Large Eddy Simulation (LES) is based on the incompressible Navier-Stokes (N-S) equations spatially filtered with the dynamic subgrid scale model. Therefore, broadband aeroacoustic noise can be predicted, because the LES resolves all eddies with scales larger than the grid scale. The central differences are used to discretize the spatial derivatives yielding overall second-order accuracy in Ansys Fluent V6.3. Initial conditions are provided by a Reynolds Averaged Navier-Stokes (RANS) simulation. The numerical method is Pressure Implicit with Splitting of Operators (PISO) algorithm on basis of the pressure-velocity formulation employing a predictor-corrector pressure scheme. The velocity $\mathrm{u}$ for the pressure far field is $8 \mathrm{~m} / \mathrm{s}$, which is typical for the flying owls, and the corresponding Reynolds number based on the chord length $\mathrm{c}$ is about $5.5 \times 10^{-4}$. The angle of attack is $0^{\circ}$, where the resulting noise source is related to the turbulent pressure fluctuations in the airfoil boundary layer. The time step $\Delta \mathrm{t}$ is $1 \times 10^{-5}$ to obtain the interesting frequency band making $\mathrm{CFL}<0.6$, calculated by $\mathrm{CFL}=\mathrm{u} \times \Delta \mathrm{t} / \Delta \mathrm{x}$. Once the flow simulation reaches a dynamically steady state, the accuracy for predicting SPL is usually dependent on the number of time steps used. Thus, the number of time steps is $1 \times 10^{6}$ for a better result.

The far-field acoustic signals are achieved by solving two-dimensional FW-H equation to gain the correct features of the noise radiated from unsteady flow around the two-dimensional bionic airfoil surface. The source correlation length for the two-dimensional simulation is $1 \mathrm{~m}$. Both the data surfaces for noise are depicted in Figure 3. The propagated noise would be overpredicted due to twodimensional method implying perfect correlation in the third dimension. However, the paper is focused on the near-field flow and the corresponding noise mechanism, so any effects associated with the overprediction of noise caused by two-dimensional approximations are obviated by the qualitative distribution trend in noise level.

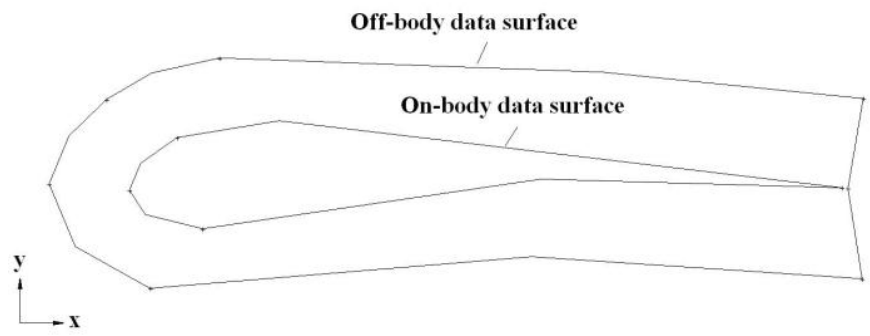

Figure 3. Schematic diagram of the data surfaces.

\subsection{Experimental setup}

Experiments are done in a low-speed wind tunnel with a cross section of $150 \times 150 \mathrm{~mm}^{2}$ and a length of $800 \mathrm{~mm}$. The wind tunnel is used as a smoke wind tunnel by installing laser sheet generator. The smoke lines are generated by engine oil dropping on an electrically heated $0.1 \mathrm{~mm}$ nichrome wire moving downstream with the air to visualize the flow field. A wing model with the chord of $150 \mathrm{~mm}$ and the span of $150 \mathrm{~mm}$ is built to avoid the spanwise flow. The freestream velocity of $8 \mathrm{~m} / \mathrm{s}$ and the 
angle of attack of $0^{\circ}$ are considered. The measurement equipment is wind tunnel LW-9117 manufactured by Long Win science and Technology Corporation.

\section{Results and discussion}

\subsection{Instantaneous flow field}

The instantaneous flow around the bionic airfoil indicates the leading-edge separation due to the adverse pressure gradient, as shown in Figure 4. After separating, the free shear layer rolls up forming a vortex by Kelvin-Helmholtz $(\mathrm{K}-\mathrm{H})$ instability. It re-energizes the low-velocity flow on the near wall, and thereby reattachment occurs with the turbulent boundary layer. This kind of separation is called the "long bubble", and its extent quickly increases as angles of attack increase until the presence of the deep stall at high angle of attack. At the downstream end of the transitional separation bubble, a large-scale vortex detaches from the bubble and moves further downstream, as depicted by Burgmann et al [18]. Simultaneously, the similar evolution is also shown on the lower surface. The reason for this is that the strong adverse pressure gradient is caused by the high curvature of the lower surface near the leading edge. In addition, the experimental flow visualized in low turbulence wind tunnel is in close agreement with the flow computed by Ansys Fluent V6.3 at the same conditions, as shown in Figure 5.

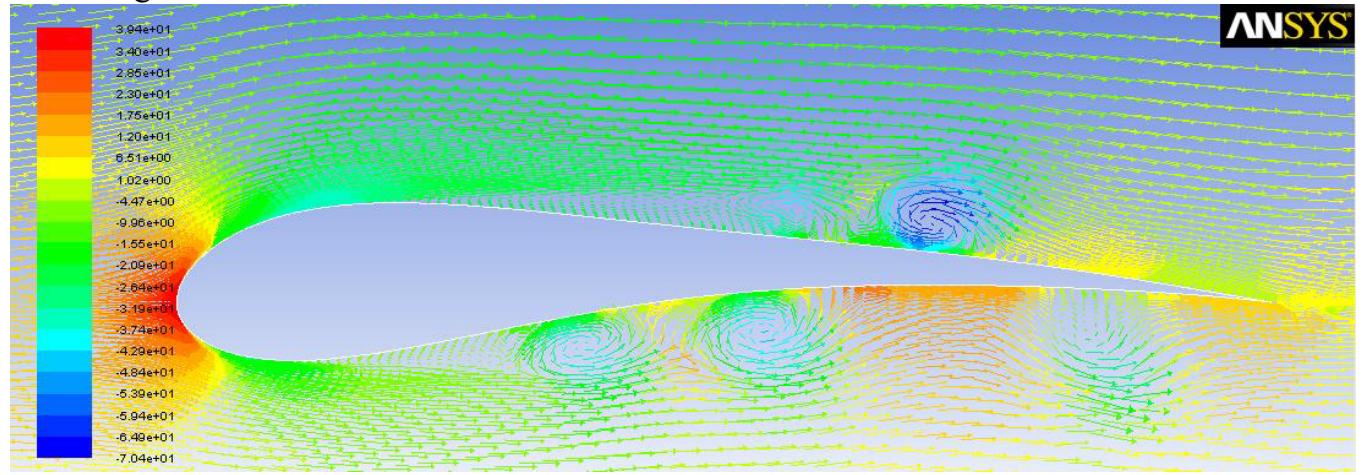

Figure 4. Distribution of velocity vector and static pressure (pa).

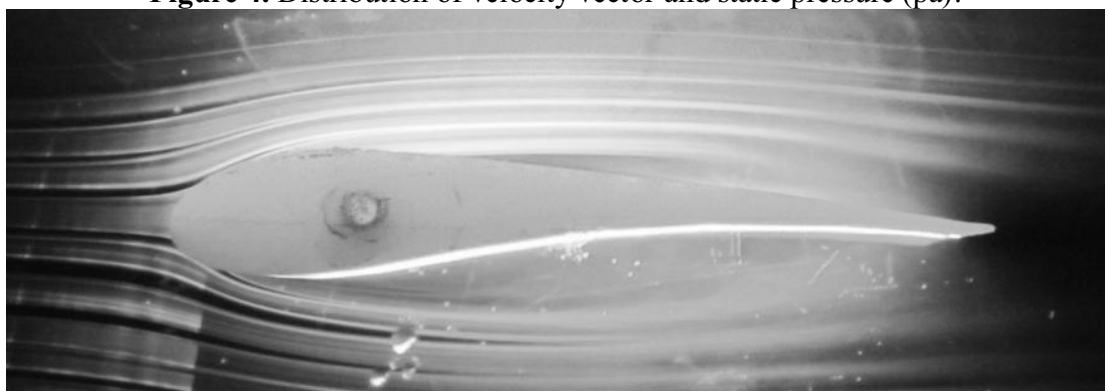

Figure 5. Flow field in wind tunnel.

The pressure coefficients $\mathrm{Cp}$ varying with the streamwise positions verifies the finding of the visualized flow field, as depicted in Figure 6 . The distribution of the upper surface shows a stable process downstream of the first peak at the chordwise position about between $\mathrm{x} / \mathrm{c}=0.2 \sim 0.5$, which corresponds to the recirculation region downstream of the leading edge in Figure 4. Subsequently, the second peak confirms the rolling up of a vortex in the bubble, and then the pressure valley indicates that the leading-edge separation is formed by the reattachment of the transitional free shear layer. Furthermore, the third (maximum) pressure peak is consistent with the large-scale vortex separated from the bubble. The following pressure fluctuation close to the trailing edge suggests the turbulent boundary layer. 


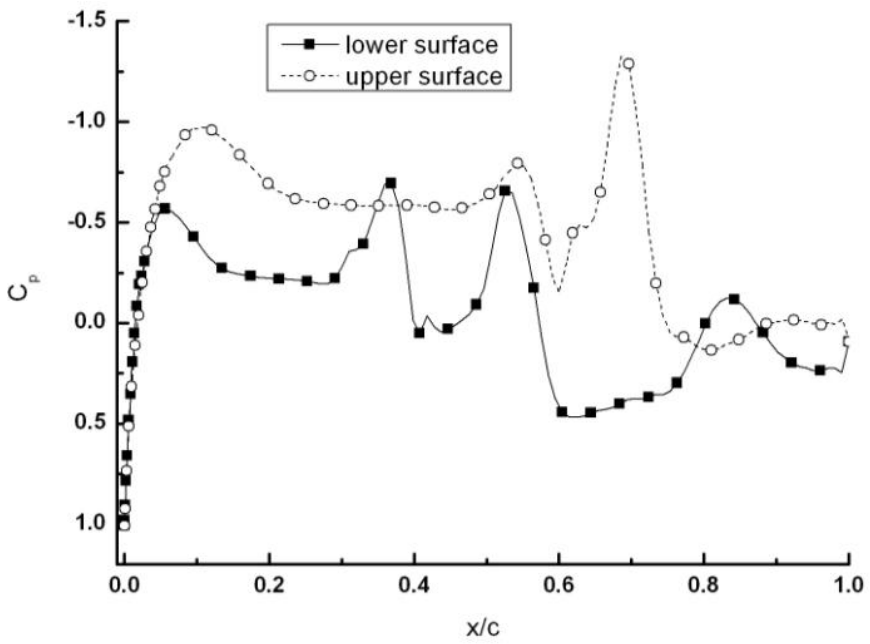

Figure 6. Distribution of pressure coefficient $\mathrm{Cp}, \mathrm{x} / \mathrm{c}$ is the normalized chordwise coordinate, where $\mathrm{x}$ is the chordwise coordinate.

The contours of vorticity also substantiate the finding of the flow field: the leading-edge separation, the roll-up process of the free shear layer, and the formation of the bubble, as shown in Figure 7. The large-scale vortex departing form the bubble and passing by the trailing edge will generate the vortex shedding responsible for the tonal loading noise. It is obvious that the K-H instability of the separated free shear layer is receptive to the acoustic perturbations caused by the trailing-edge vortex shedding and the process can produce an aeroacoustic feedback loop. Interesting, the turbulent boundary layer, which reattaches on the wall, will cause the broadband scattering noise due to the interaction of the turbulent eddies within the boundary layer with the sharp trailing edge of the bionic airfoil. This can be used to explain the noise mechanism of natural owl wings at the low Reynolds numbers.

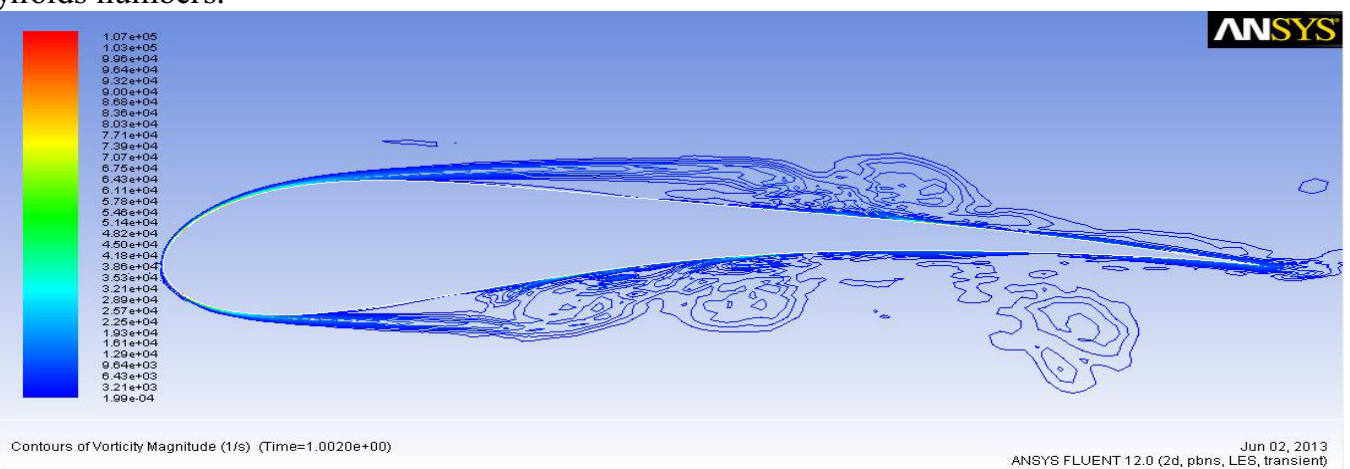

Figure 7. Contours of vorticity (1/s).

\subsection{Acoustic field}

Figure 8 shows a comparison of the acoustic spectra obtained at a distance of 10c directly under the leading edge for cases, in which the on-body data surface and the off-body data surface are used to compute the far-field noise. The humps agree well with the broadband noise nature of owls on the approach. The lack of the strong peak means that vortex-shedding noise is less than turbulent boundary layer trailing-edge noise that is the dominant source in the current simulations. The significant discrepancy of the SPLs between both the cases suggests that the on-body data surface can only capture the scattering source caused by wall pressure fluctuation in the turbulent boundary layer. In contrast, the off-body can also consider quadrupole effect of turbulence in the boundary layer by the permeable data surface. In particular, the collapse depicted in the SPLs is expected around $2 \mathrm{k} \mathrm{Hz}$, 
because landing owls can dramatically decrease noise above $2 \mathrm{k} \mathrm{Hz}$ [2 4]. This indicates that the acoustic prediction is believable, although amplitudes for the on-body data surface are significantly lower than those for off-body data surface.

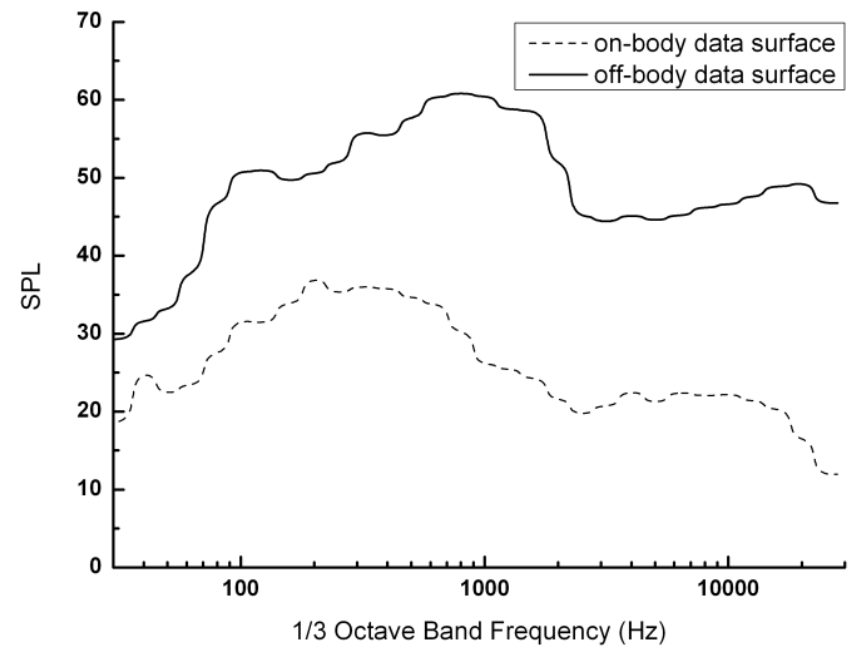

Figure 8. Sound pressure level at the observer position displaced 10c directly under the leading edge.

\section{Conclusion}

The unsteady simulation of the bionic airfoil is completed in order to investigate the noise related to owl wings. The flow field around the airfoil shows that the leading-edge boundary layer on the surface is separated, and later the free shear layer rolls up forming a vortex due to K-H instability. As a result, a bubble comes into being by the reattachment of the transitional free shear layer. The resulting turbulent boundary layer is responsible for the noise mechanism of owl wings. Furthermore, a largescale vortex detaches from the bubble and drifts downstream. Thus, the aeroacoustic feedback loop is achieved by the interaction of trailing-edge vortex shedding with the free shear layer.

The flow from the unsteady LES calculation is used to provide the necessary information on the on-body and off-body data surfaces. The preliminary results indicate the broadband nature of the noise, which is consistent with the scattering caused by flying owls on the approach. Compared to the on-body data surface applied at the bionic airfoil, the off-body data surface, which contains the dominant vortexes on the basis of vorticity field, can account for the non-linear effect of sound scattering by interaction between turbulence and sharp trailing edge. In addition, both the spectra generated by the airfoil dramatically decrease around $2 \mathrm{k} \mathrm{Hz}$, as in the spectra of silent owl wings. This similarity suggests that the simulation for the unsteady flow around the bionic airfoil can predict the scattering noise mechanism generated by natural owl wings.

\section{Acknowledgements}

The authors would like to thank the Key Laboratory of Bionic Engineering for their assistance with the specimen and the equipments. This research is supported by the National Natural Science Fund of China (No. 51505182) and Jilin Scientific and Technological Development Program (No. 20160520065JH).

\section{References}

1. R.A. Kroeger, H.D. Gruschka, T.C. Helvey, Air Force Flight Dynamics Laboratory Technical Report Low speed aerodynamics for ultra-quiet flight., 71-75 (1971). 
2. G.M. Lilley, A study of the silent flight of the owl. AIAA Paper, 98-2340 (1998).

3. G.M. Lilley, The Prediction of Airframe Noise and Comparison with Experiment. Journal of Sound and Vibration, 239 (4), 849-859 (2001).

4. D.P. Lockard, G.M. Lilley, The Airframe Noise Reduction Chanllenge. NASA/TM-2004-213013 (2004).

5. R.R. Graham, The silent flight of owls. J. R. Aeronaut., 38, 837-843 (1934).

6. M.S. Howe, Aerodynamic noise of a serrated trailing edge. J. Fluids and Structures, 5, 33-45 (1991).

7. M.S. Howe, On the added mass of a perforated shell, with application to the generation of aerodynamic sound by a perforated trailing edge. Proc. R. Soc. Lond. A, 365, 209-233 (1979).

8. S. Oerlemans, M. Fisher, T. Maeder, K. Kogler, Reduction of wind turbine noise using optimized airfoils and trailing edge serrations. AIAA J., 47(6):1470-1481 (2009).

9. M. Herr, New Results in Numerical and Experimental Fluid Mechanics V: Experimental Study on Noise Reduction through Trailing Edge Brushes, Springer, Berlin Heidelberg, 365-372 (2006).

10. M. Herr, W. Dobrzynski, Experimental investigations in low-noise trailing-edge design. AIAA J., 43(6), 1167-1175 (2005).

11. M. Herr, On the design of silent trailing edges. New Res. in Num. and Exp. Fluid Mech. VI, NNFM 96, 96, 430-437 (2007).

12. T.Geyer, E. Sarradj, C. Fritzsche, Porous airfoils: Noise reduction and boundary layer effects. AIAA Paper, 2009-3392 (2009).

13. T. Geyer, E. Sarradj, C. Fritzsche, Measurement of the noise generation at the trailing edge of porous airfoils. Exp. in Fluids, 48, 291-308 (2010).

14. T.S. Liu, K. Kuykendoll, R. Rhew, S. Jones, Avian wing geometry and kinematics, AIAA J., 44, 954-963 (2006).

15. S. Klan, T. Bachmann, M. Klaas, H. Wagner, W. Schroder, Experimental analysis of the flow field over a novel owl based airfoil. Exp. in Fluids, 46, 975-989 (2009).

16. R.A. Kroeger, H.D. Gruschka, T.C. Helvey, Low speed aerodynamics for ultra-quiet flight. Air Force Flight Dynamics Laboratory Technical Report, 71-75 (1971).

17. C. Ge, Z. Zhang, P. Liang, C. Zhang, L. Ren, Prediction and control of trailing edge noise based on bionic airfoil. SCIENCE CHINA Technological Sciences E, 57(7), 1462-1470 (2014).

18. Burgmann, S., Dannemann, J., Schroder W. Time-resolved and volumetric PIV measurements of a transitional separation bubble on an SD7003 airfoil. Exp. in Fluids, 44 (4), 609-622 (2008). 